\title{
High impedance fault detection in distribution system
}

\author{
Kavaskar Sekar ${ }^{1}$, Nalin Kant Mohanty ${ }^{2}$ \\ ${ }^{1}$ Department of Electrical and Electronics Engineering, Panimalar Engineering College, India \\ ${ }^{2}$ Department of Electrical and Electronics Engineering, Sri Venkateswara College of Engineering, India
}

\begin{tabular}{l} 
Article Info \\
\hline Article history: \\
Received Aug 30, 2018 \\
Revised Mar 20, 2019 \\
Accepted Apr 17, 2019 \\
\hline Keywords: \\
Discrete wavelet transform \\
High impedance fault \\
Neural networks \\
Non-linear load
\end{tabular}

Non-linear load

\begin{abstract}
High impedance faults (HIFs) present a huge complexity of identification in an electric power distribution network (EPDN) due to their characteristics. Further, the growth of non-linear load adds complexity in HIF detection. One primary challenge of power system engineers is to reliably detect and discriminate HIFs from normal distribution system load and other switching transient disturbances. In this study, a novel HIF detection method is proposed based on the simulation of an accurate model of an actual EPDN study with real data. The proposed method uses current signal alone and does not require voltage signal. Wavelet transform (WT) is used for signal decomposition to extract statistical features and classification of HIF into Non-HIF (NHIF) by Neural Networks (NNs). The simulation study of the proposed method provides good, consistent and powerful protection for HIF.
\end{abstract}

Copyright (C) 2019 Institute of Advanced Engineering and Science. All rights reserved.

\section{Corresponding Author:}

Kavaskar Sekar, Department of Electrical and Electronics Engineering, Anna University, Guindy, Chennai, Tamil Nadu 600025, India. Email:kavaskarsekar@gmail.com

\section{INTRODUCTION}

The majority of electric power distribution lines in India are overhead lines. These lines are subject to disturbance such as an energized conductor falls to the ground or connected to high impedance objects due to dissimilar environments. This condition is called High impedance fault (HIF), and it draws little fault current which is less compared to the threshold values of conventional protection means. Hence, some of these HIFs may not be detected. Further, threshold values cannot set as lower values, since the normal load distribution will lead to nuisance tripping. Besides, HIFs are accompanied by electric arcs. These arcs produce an asymmetric, unpredictable, and random current signal. Most of the electric power distribution networks (EPDN) are in close proximity to the thickly populated area. If EPDN is operated with HIF leads to endangering the human lives and their properties or material goods. Therefore, with the goal of increasing the performance and the safety of EPDN, several papers have been published in the past few decades to solve HIF problem is well documented in [1]. The redefining process of detection yet to be completed due to an increase in non-linear loads (NLLs).

A method based on harmonic content presented in $[2,3]$. The setback of such method is to set threshold values which reduces the performance of the method. Time-frequency analysis [4, 5] produces better performance in HIF detection. The modified Fast Fourier Transform (FFT) method employs the relative relation between the third, fifth and seventh harmonic current in [6]. The presence of NLLs taken into account and reliable detection shown in the system studied. However, the percentage of false tripping and computational work is an obstacle for practical implementations. Time-domain based method called mathematical morphology [7, 8] is used for detection of HIF, which is good for a balanced system. When the system is unbalanced, these methods show less performance.

Wavelet Transform (WT) has been used in HIF detection. WT is analyzing the signal with frequency component and their position in time. More than 20 years such methods used in protection 
applications. The algorithm in [9] uses dynamic features extracted by stationary WT and a support vector machine based decision-making system. Arcing currents related to different high impedance surface are generated in the laboratory setup, and these signals are decomposed using Discrete Wavelet Transform (DWT) [10]. The authors used DWT to examine high- and low-frequency voltage components at different points [11] and an average of absolute difference of extracted voltage signal [12] of EPDN. In [13] voltage and current signals are used to detect and locate HIF respectively. This method uses the absolute sum of high-frequency components for both detection and location. Two different HIF detection scheme are discussed in [14], both utilizing WT decomposition. In the first method, principal component analysis (PCA) for feature reduction and Neural Network $(\mathrm{NN})$ for feature classification. In the second method, a Genetic Algorithm (GA) for feature reduction and Bayes clasifier for classification. The change in current signal created by HIF and other transient events has been used in [15] with NLLs in the system. A DWT used to decompose the current signals and extracts features to train NNs. An evolving NN [16] and continually online trained NN method [17] was shown as the proper approach to HIF detection since it is a time-varying problem. A DWT with data mining based classification in [18].

These research articles disclose useful properties and different detection methods of HIF. However, the majority of the above-mentioned articles fail to consider NLLs except in $[6,15,18]$. These three articles are showing less variation in NLLs. Since NLLs such as television, computer, fluorescent lamps, etc., constantly increasing year by year in the distribution grids and NLLs and HIFs characteristics have closely resembled each other, which will make the existing methods less effective. Hence, an enhanced detection method for HIFs with a massive variation of NLLs is Proposed.

The rest of the paper has been prepared as follows. Section 2 explains the test system and also the characteristics of HIF model engaged for simulation. The proposed methodology has been thoroughly discussed in Section 3, including the decomposition of a signal, feature extraction, feature selection, and classification. Section 4 has results and discussion and the paper concluded with the main highlights of the work in section 5 .

\section{TEST SYSTEM}

\subsection{System studied}

The proposed method is verified through an actual EPDN in India (Chennai) shown in Figure 1 was modeled with a sim-power-system block set (MATLAB). The system data are given in [18]. Distribution lines are modeled as lumped parameters. Busbar input is modeled by Thevenin's voltage and equivalent impedance.

\subsection{HIF model and characteristics}

The HIF Emanuel's model [4] shown in Figure 2 used in this work. The HIF current signal during different parameters of the model is shown in Figure 3, which shows the non-linear behaviour, asymmetry in the signal, fewer current and random behavior of fault current. This is due to low- and high-frequency content of the signal is changing with time and hence the signal is termed as a non-stationary signal.

\subsection{Test conditions}

For the classification HIF and non-HIF (NHIF), a total of 540 HIF and 460 NHIF cases are described in Table 1.

\section{PROPOSED METHODOLOGY}

The proposed HIF detection approach is shown in Figure 4. The process starts with the simulation of EPDN and accessing current signal. The DWT decomposes the current signal and features such as energy, entropy, skewness, sum, standard deviation, and kurtosis are extracted. These features are training two layer feed forward NNs to classify the case belongs to HIF or NHIF.

\subsection{Signal decomposition using wavelet transform}

Wavelet is an effective tool to analyze non-stationary signal with the capability of multiple resolutions in time and frequency. DWT is a flexible signal processing method broadly used in power system engineering. DWT has been successful in stability analysis of power system engineering. The mathematical equation for DWT represented in (1).

$$
D W T_{(m, n)}=\frac{1}{\sqrt{2^{m}}} \sum \mathrm{x}(\mathrm{k}) \psi\left(\frac{n-k 2^{m}}{2^{m}}\right)
$$

Int. J. of Adv. in Appl. Sci. Vol. 8, No. 2, June 2019: 95 - 102 
where $\mathrm{x}(\mathrm{k})$ is the discrete signal in terms of coefficients.

$\psi($.$) is mother wavelet, \mathrm{m}$ and $\mathrm{n}$ are time scale parameters.

$\mathrm{k}$ is discrete time of coefficients.

$2^{\mathrm{m}}$ is scaling parameter.

$\mathrm{k} 2^{\mathrm{m}}$ is shifting parameter.

$\frac{1}{\sqrt{2^{m}}}$ is the energy normalization component.

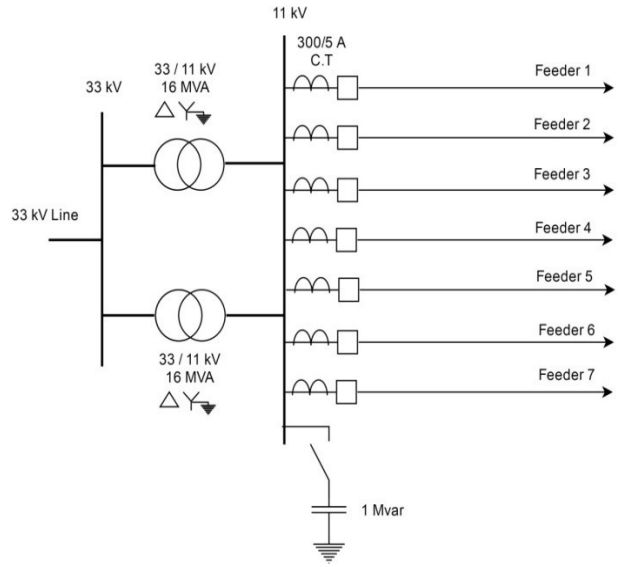

Figure 1. EPDN studied

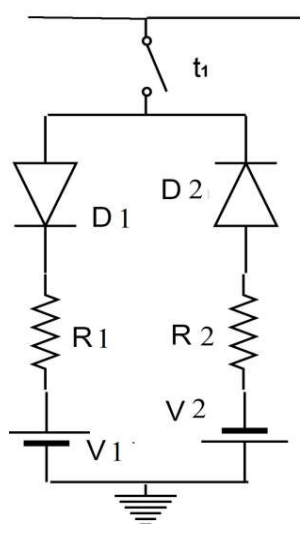

Figure 2. HIF model

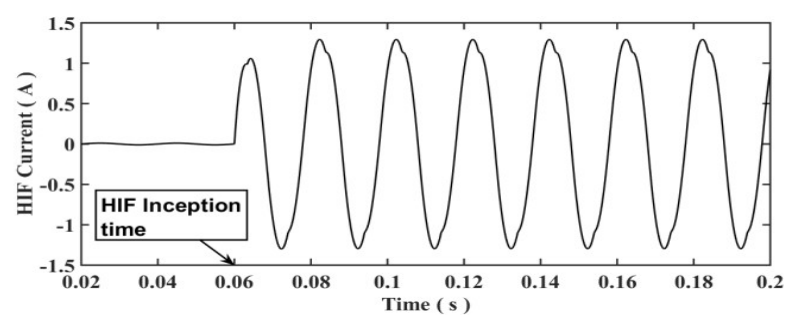

(a)

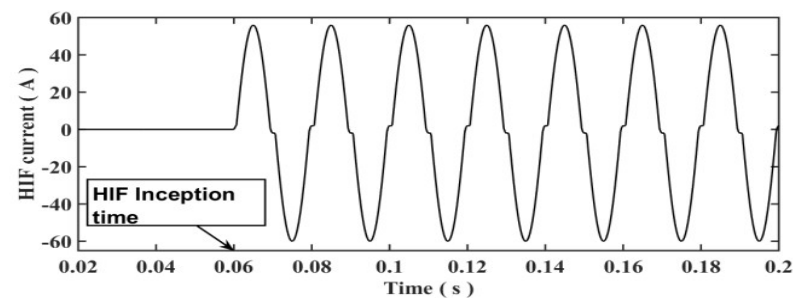

(b)

Figure 3. HIF current waveform

(a) $\mathrm{R} 1=10000 \Omega, \mathrm{R} 2=9000 \Omega, \mathrm{V} 1=5000 \mathrm{~V}, \mathrm{~V} 2=5050 \mathrm{~V}$.

(b) $\mathrm{R} 1=150 \Omega, \mathrm{R} 2=160 \Omega, \mathrm{V} 1=2000 \mathrm{~V}, \mathrm{~V} 2=2050 \mathrm{~V}$.

Low pass filters (LPF) and high pass filters (HPF) is used in DWT to decompose a signal into a lowfrequency and high-frequency component. The decomposition process starts by passing a signal through LPF and HPF. The LPF produces an approximate coefficient which has high scaled and low-frequency decomposition. In contrast, the HPF produces detailed coefficient which has low scaled and high-frequency 
decomposition. At once the first level of decomposition is completed, the sampling frequency is reduced by half of its value. To calculate the next DWT level, LPF output (approximation) is decomposed. The Daubechies basis db4, five level decomposition, and the sampling rate of $25.6 \mathrm{kHz}$ with the data window length of three cycles are chosen in this work.

Table 1. Test conditions

\begin{tabular}{lll}
\hline Event & Simulated conditions & \\
\hline HIF & $\begin{array}{l}\text { Resistances varied from } 150 \Omega \text { to } 12 \mathrm{k} \Omega \text { and DC voltage from } 1.5 \mathrm{kV} \text { to } 10.5 \mathrm{kV} \\
\text { randomly } \\
\text { Load switching }\end{array}$ & $\begin{array}{l}\text { Changes in load level: } 0 \%-25 \%, 25 \%-50 \%, 50 \%-75 \%, 75 \%-100 \% \text { and } 100 \% \\
\text { both forward and reverse conditions. } \\
\text { The ratio of linear to non-linear load changes } 1 \text { to } 0.5 \text { in steps of } 0.05 \text { both in forward and } \\
\text { NLL switching } \\
\text { reverse conditions. }\end{array}$ \\
$\begin{array}{l}\text { Capacitor } \\
\text { Switching } \\
\text { Inrush current }\end{array}$ & No load switching of $33 / 11 \mathrm{kV}$ transformer & 216 \\
\hline
\end{tabular}

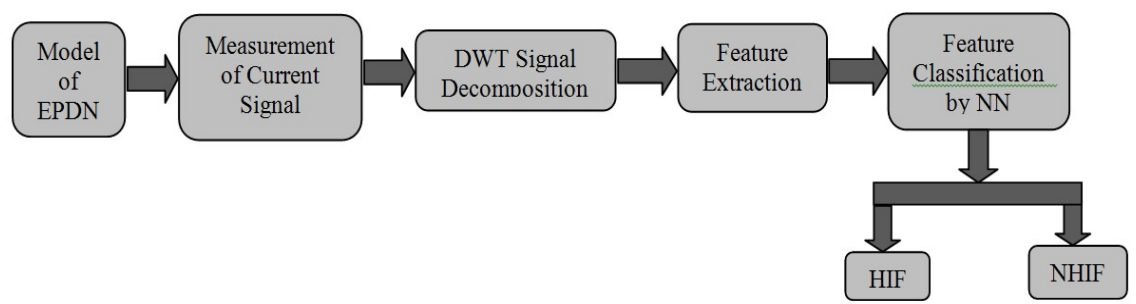

Figure 4. The proposed HIF detection approach

The decomposition of phase a current due to NLLs and capacitance switching at inception time of $0.065 \mathrm{~s}$ is shown in Figure 5 and Figure 6 . It is found that the behavior of both NLL and capacitance has low and high-frequency parts in all wavelet detail coefficient D5 to D1. The decomposition of phase a current during HIF is shown in Figure 7. A small magnitude of change in frequency component is present since the initiation of HIF in detail coefficient D5 alone.

\subsection{Selection of wavelet coefficient}

From the DWT analysis of the current signal, there were one approximation coefficient (A5) and five detailed coefficients (d1-d5) acquired. The comparison of different wavelet detail and approximation coefficient has been made to select wavelet coefficient for HIF Detection. It is observed that detail coefficients $\mathrm{d} 4$ and $\mathrm{d} 5$ offers diverse characteristics during different power system events and hence selected for feature extraction.

\subsection{Features using wavelet coefficients $d 4$ and $d 5$}

Six different features were estimated using wavelet coefficients $\mathrm{d} 4$ and $\mathrm{d} 5$ are described below:

a. Standard deviation: It represents the deviation of a signal from its mean.

b. Energy: The total energy content of the current signal.

$$
\text { Energy }=\frac{1}{\mathrm{~T}} \int_{0}^{\mathrm{T}} \mathrm{i}^{2}(\mathrm{t}) \mathrm{dt}
$$

c. Kurtosis: Bigger kurtosis point represents more outlier in the signal.

$$
\begin{aligned}
& \text { Kurtosis }=\left\{\frac{\mathrm{n}(\mathrm{n}+1)}{(\mathrm{n}-1)(\mathrm{n}-2)(\mathrm{n}-3)} \sum \frac{(\mathrm{x}-\overline{\mathrm{x}})^{4}}{\sigma^{4}}\right\}-\frac{3(\mathrm{n}-1)^{2}}{(\mathrm{n}-2)(\mathrm{n}-3)} \\
& \sigma \text { - Standard deviation, } \bar{x}-\text { Mean, } \mathrm{n}-\text { no of sample data }
\end{aligned}
$$

d. Skewness: It is a measure of the irregularity of the probability distribution about its mean.

Int. J. of Adv. in Appl. Sci. Vol. 8, No. 2, June 2019: 95 - 102 


$$
\text { Skewness }=\left\{\frac{\mathrm{n}}{(\mathrm{n}-1)(\mathrm{n}-2)} \sum \frac{(\mathrm{x}-\overline{\mathrm{x}})^{3}}{\sigma^{3}}\right\}
$$
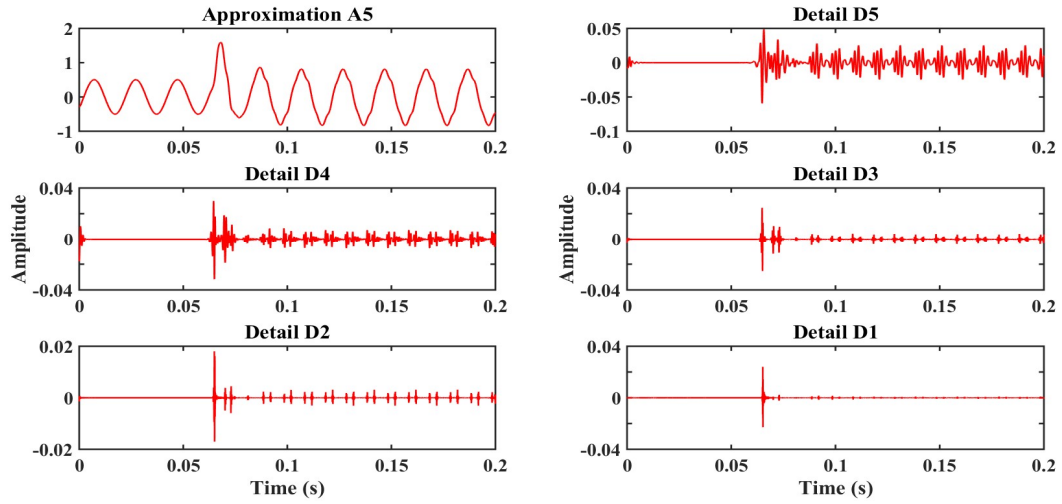

Figure 5. DWT of current signal under non-linear load switching
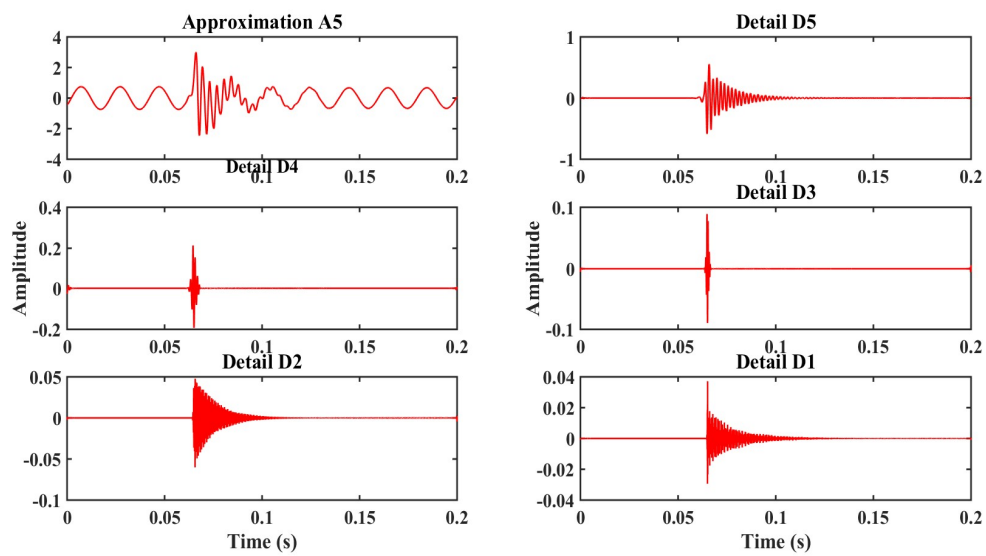

Figure 6. DWT of current signal under capacitance switching
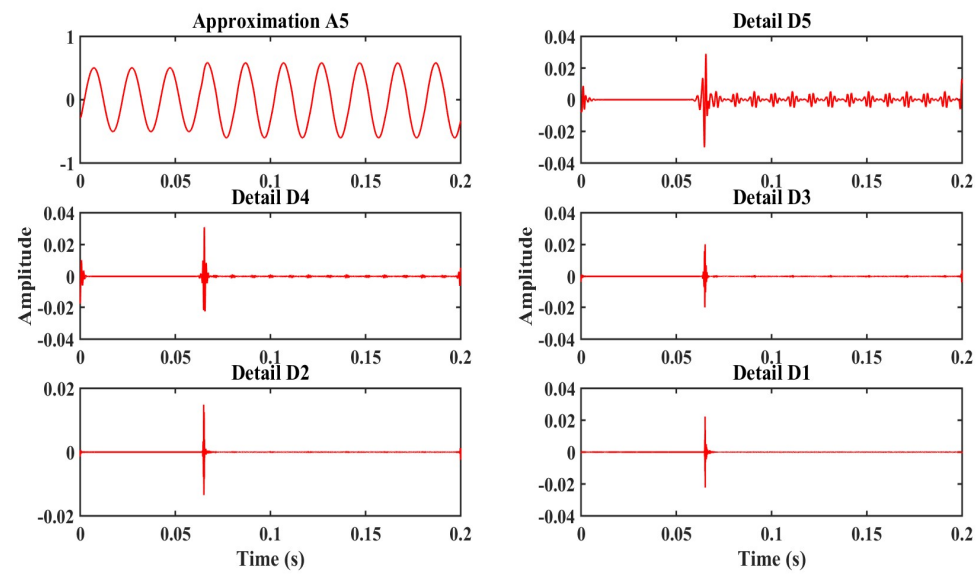

Figure 7. DWT of current signal under HIF 
e. Entropy: It gives complexity of the signal.

$$
\text { Entropy }=-\sum \mathrm{p}_{\mathrm{j}} \log \mathrm{p}_{\mathrm{j}}
$$

Where $\mathrm{p}_{\mathrm{j}}$ is an energy probability distribution of WT detail coefficient (d4 and d5)

f. Sum: Sum of all points of signal.

\subsection{NN implementation}

The NN is a powerful tool used in pattern recognition and classification [19]. Consequently, the proposed work uses $\mathrm{NN}$ to learn the input and output relationship from feature input vector. As mentioned in the feature extraction process, the input to NN comprises 12 elements ( 6 features of detail coefficients $\mathrm{d} 4$ and d5). The output layer has two neurons i.e. either HIF or NHIF and 10 hidden layers. A two-layer feedforward NN has been used in this work as shown in Figure 8. The sigmoid activation function used for hidden layer and softmax for the output layer. The NN is trained with scaled conjugate back-propagation that updates weight and bias values. Also, the number of epochs was determined by experimentation. The number of epochs for the training was set around 1000 and a mean squared error rate of $\mathrm{e}^{-03}$ was used [20]. The dataset is randomly divided into three parts: a training data set of $70 \%$, validation of $15 \%$ dataset, and the remaining $15 \%$ for testing.

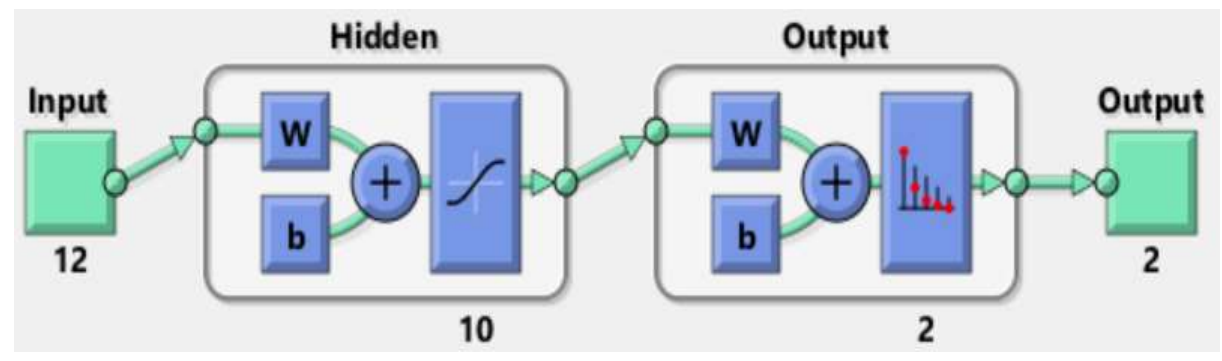

Figure 8. NN model for HIF detection

\section{RESULTS AND DISCUSSIONS}

A set of 1000 features obtained by changing the simulation conditions as given in Table 1 . Thus a wide range of studies has been carried out to demonstrate the proposed method. Conversely, it is not possible to report all information because of space constraint. The proposed method is evaluated through the following three parameters:

- Dependability: Predicted HIF against total HIF conditions.

- $\quad$ Security: Predicted Non-HIF against total non-HIF conditions.

- Accuracy: Actual predicted against the total number of conditions considered.

- $\quad$ Speed: One cycle power frequency current / No of cycles that it takes to detect the fault.

The confusion matrix of the NN during training, validation, and testing are presented in Figure 9. During training, all 370 HIF test cases are correctly classified to produce dependability of $100 \%$. Two NHIF test case is misclassified as HIF to produce $99.4 \%$ security with the overall accuracy of $99.7 \%$. During validation of 150 cases, dependability observed as $100 \%$, and $97 \%$ of security with the overall accuracy of 98.7\%. Finally, during testing of 150 cases, all HIF cases predicted correctly and only one NHIF cases are classified as HIF and produce dependability, security, and accuracy as $100 \%, 98.4 \%$, and $99.3 \%$ respectively. It is very clear from the confusion matrix that the method has an excellent detection rate. Nevertheless, there was a huge amount of non-linear load variation, all the HIF cases are detected.

Power system more vulnerable to noise, it is necessary to investigate the proposed method with different Signal to Noise Ratio (SNR). Table 2 compares the performance under noisy atmosphere. It noted that the performance is similar to the one observed in normal case for $10 \mathrm{~dB}$ of SNR on the signal. For $20 \mathrm{~dB}$ of SNR, HIF detection rate alone same. However, the accuracy and security are reduced to $98 \%$ and $95.16 \%$ respectively. Additional SNR reduces all the three performance indices. Though the accuracy of $20 \mathrm{~dB}$ is less, the HIF detection rate is excellent, and hence the proposed method is suitable for SNR of 20 or less.

Int. J. of Adv. in Appl. Sci. Vol. 8, No. 2, June 2019: 95 - 102 

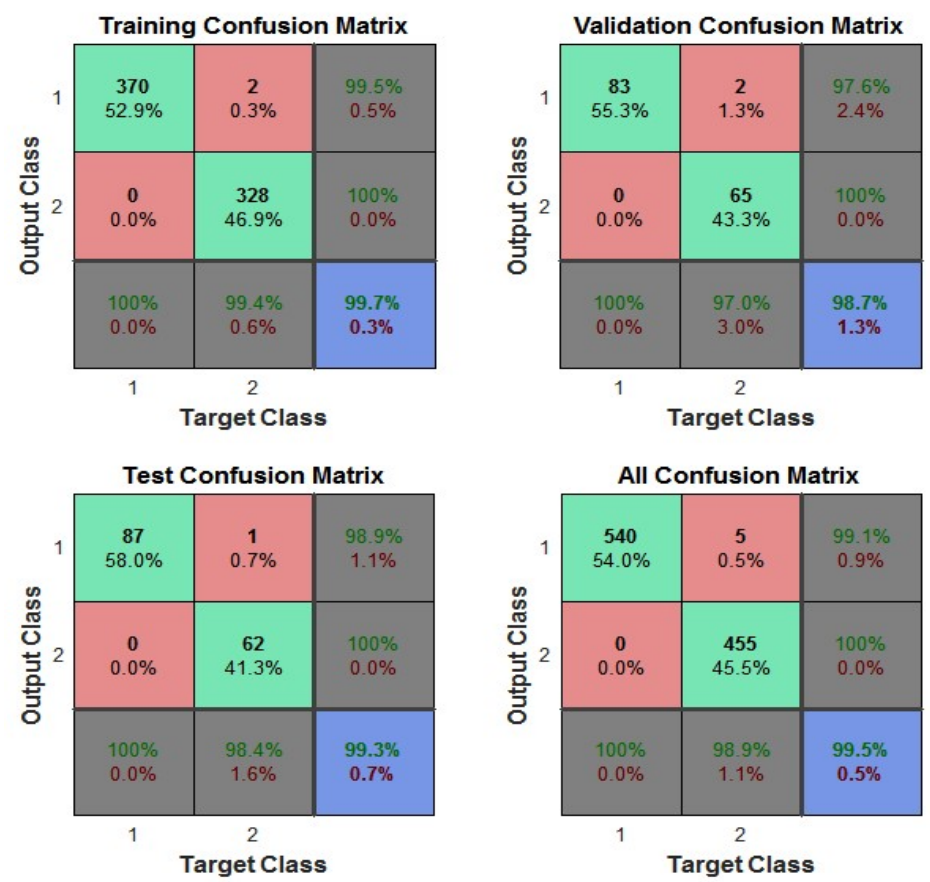

Figure 9. The confusion matrix of NNs

Table 3 shows the impact of the sampling rate of the proposed work. There was substantial upgrading demonstrated by the proposed methodology with sampling rate from 256 to 512 . The detection rate diminishes as the sampling rate value decreases. Hence, the proposed scheme is better with 512 samples per cycle.

Finally, the performance of the proposed HIF approach is compared with previously published articles as presented in Table 4. It is observed that all three performance indices are better than [4, 7, 10, 14]. The speed of detection is compared with the references mentioned in Table 4 . The speed of detection is low compared to [4] and as good as with other references listed in Table 4. However, it is noted that HIFs are low current, during which the distribution network are not in stress. As a result, the HIF detection speed is not a critical concern. In other hand, the security index of the HIF detection algorithm is important as well as its dependability index.

Table 2. Impact of SNR on the proposed method

\begin{tabular}{cccc}
\hline Performance indices & $10 \mathrm{~dB}$ & $20 \mathrm{~dB}$ & $30 \mathrm{~dB}$ \\
\hline Accuracy & $99.33 \%$ & $98.00 \%$ & $93.33 \%$ \\
Security & $98.38 \%$ & $95.16 \%$ & $88.70 \%$ \\
Dependability & $100 \%$ & $100 \%$ & $96.59 \%$ \\
\hline
\end{tabular}

Table 3. Impact of sampling rate on the proposed method

\begin{tabular}{cccc}
\hline Performance indices & 512 & 420 & 256 \\
\hline Accuracy & $99.33 \%$ & $96.00 \%$ & $86.66 \%$ \\
Security & $98.38 \%$ & $95.16 \%$ & $80.64 \%$ \\
Dependability & $100 \%$ & $98.88 \%$ & $90.90 \%$ \\
\hline
\end{tabular}

Table 4. Comparison of the proposed method with previously published work

\begin{tabular}{ccccc}
\hline Method & Accuracy (\%) & Security (\%) & Dependability (\%) & Speed \\
\hline Ref [4] & 93.6 & 81.5 & 100 & 1 \\
Ref [7] & 97.3 & 96.3 & 98.3 & 0.04 \\
Ref [14] & 96 & 100 & 90 & 0.25 \\
Ref [10] & - & 68 & 72 & 0.33 \\
The proposed method & 99.33 & 98.38 & 100 & 0.33 \\
\hline
\end{tabular}

High impedance fault detection in distribution system (Kavaskar Sekar) 


\section{CONCLUSION}

In this paper, a new methodology based on the WT NNs is presented. The proposed method efficiently distinguishing HIF form other normal patterns. The proposed method comprehensively studied an actual electric power system with real data using MATLAB with a huge variation in power system operating conditions. The method has an exceptional detection rate even under wide variation in NLLs. This indicates that the proposed scheme is highly consistent and secure detection for HIFs with a dependability index of $100 \%$.

\section{REFERENCES}

[1] Ghaderi, H., Mohammadpour, HA, Ginn, HL, and Shin, YJ, "High Impedance Fault detection -a review," Electr. Power Syst. Res, vol. 146, pp. 376-388, 2017.

[2] Emanuel, D., Cyganski, J., Orr S., Shiller, and E. Gulachenski, "High impedance fault arcing on sandy soil in 15 $\mathrm{kV}$ distribution feeders: contributions to the evaluation of the low frequency spectrum," IEEE Trans. Power Deliv, vol. 5(2), pp. 676-686, 1990.

[3] Esmaeil, D. and Jamal, B., "High Impedance Fault Detection in Power Distribution Networks with Use of Current Harmonic-Based Algorithm," Indonesian Journal of Electrical Engineering and Informatics, vol. 3(4), pp. 216-223, 2015.

[4] Ghaderi, H., Mohammadpour, HA, Ginn, HL, and Shin, YJ, "High impedance fault detection in distribution network using time-frequency based algorithm," IEEE Trans. Power Deliv, vol 30(3), pp. 1260-1268, 2015.

[5] Samantaray, SR., Panigrahi, BK., and Dash, PK., "High-impedance fault detection in power distribution networks using time-frequency transform and probabilistic neural network," IET Gener. Transm. Distrib, vol. 2(2), pp. 261-270, 2008.

[6] Soheili, A., Sadeh, J., and Bakhshi, R., "Modified FFT-based high-impedance fault detection technique considering distribution non-linear loads: simulation and experimental study," International Journal of Electrical Power and Energy Systems, vol. 94, pp. 124-140, 2018.

[7] Sarlak, M. and Shahrtash, SM., "High-impedance fault detection using combination of multilayer perceptron neural networks based on multi resolution morphological gradient features of current waveform," IET Gener. Transm. Distrib, vol 5(5), pp. 588-595, 2011.

[8] Kavaskar, S. and Mohanty NK., "Combined mathematical morphology and data mining based high impedance fault detection," Energy Procedia, vol. 117, pp. 417-423, 2017.

[9] Hamid Mortazavi, S., Moravej, Z., and Mohammad, SS., "A hybrid method for arcing faults detection in large distribution networks," International Journal of Electrical Power and Energy Systems, vol. 94, pp. 141-150, 2018.

[10] Jichao, C., Toan, P., Trevor, B., Eliathambi, A., and Daming, Z., "Detection high impedance fault using current transformer for sensing and identification based method on features extracted using wavelet transform," IET Gener. Transm. Distrib, vol 10(12), pp. 2290-2298, 2016.

[11] Santos, WC, Lopes, FV., Brito, NSD., and Souza, BA., "High-impedance fault identification on distribution networks" IEEE Trans. Power Del, pp. 32(1), pp. 23-32, 2017.

[12] Bakar, AHA., Ali, M., Tan, C., Mokhlis, H., Arof, H., and Illias, H., "High-impedance fault location in $11 \mathrm{kV}$ underground distribution systems using wavelet transforms" International Journal of Electrical Power and Energy Systems, vol. 55, pp. 723-730, 2014.

[13] Mahari, H. and Seyed, "High impedance fault protection in transmission lines using a WPT-based algorithm," International Journal of Electrical Power and Energy Systems, vol. 67, pp. 537-545, 2015.

[14] Sedighi, AR., Haghifam, MR., and Malik, OP., "Soft computing application in high impedance fault detection in distribution system," Electr. Power Syst. Res, vol. 76(1-3), pp. 136-144, 2005.

[15] Baqui, I, Zamora, I., Mazón, J., and Buigues, G., "High impedance fault detection methodology using wavelet transform and artificial neural networks," Electr. Power Syst, Res, vol. 81(7), 1325-1333, 2011.

[16] Sergio, S., Pyramo, C., Maury, G., Alcyr, L., Franciele, A., and Daniel, L., "High impedance fault detection in power distribution systems using wavelet transform and evolving neural network," Electric Power Systems Research, vol. 154, pp. 474-483. 2018.

[17] Patrick, EF, Adriano, PM., Jean, PR., and Ghendy, C. "Non-linear high impedance fault distance estimation in power distribution systems: A continually online-trained neural network approach," Electric Power Systems Research, vol. 157, pp. 20-28, 2018.

[18] Kavaskar, S., Mohanty, NK, and Sahoo, AK. "High-impedance fault detection using wavelet transform," IEEE conference on 2018 Technologies for Smart-City Energy Security and Power, pp. 1-6, Bhubaneswar, 2018.

[19] Bishop, CM, Neural Networks for Pattern Recognition. Oxford University Press, 1996.

[20] Adewole, AC., Tzoneva, R., Shaheen, B., "Distribution network fault section identification and fault location using wavelet entropy and neural networks," Applied Soft Computing, vol 46, pp. 296-306, 2016.

Int. J. of Adv. in Appl. Sci. Vol. 8, No. 2, June 2019: 95 - 102 